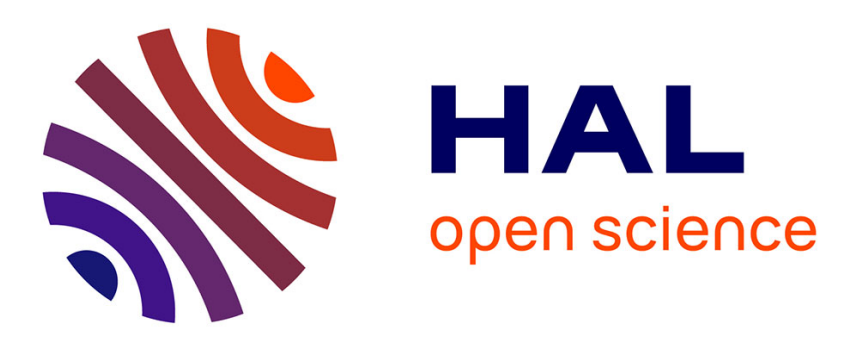

\title{
Enhancing positioning accuracy through direct position estimators based on hybrid RSS data fusion
}

\author{
Mohamed Laaraiedh, Stéphane Avrillon, Bernard Uguen
}

\section{To cite this version:}

Mohamed Laaraiedh, Stéphane Avrillon, Bernard Uguen. Enhancing positioning accuracy through direct position estimators based on hybrid RSS data fusion. Vehicular Technology Conference, 2009. VTC Spring 2009. IEEE 69th, Apr 2009, Barcelona, Spain. pp.1 - 5. hal-00379638

\section{HAL Id: hal-00379638 \\ https://hal.science/hal-00379638}

Submitted on 29 Apr 2009

HAL is a multi-disciplinary open access archive for the deposit and dissemination of scientific research documents, whether they are published or not. The documents may come from teaching and research institutions in France or abroad, or from public or private research centers.
L'archive ouverte pluridisciplinaire HAL, est destinée au dépôt et à la diffusion de documents scientifiques de niveau recherche, publiés ou non, émanant des établissements d'enseignement et de recherche français ou étrangers, des laboratoires publics ou privés. 


\title{
Enhancing positioning accuracy through direct position estimators based on hybrid RSS data fusion
}

\author{
M.Laaraiedh, S.Avrillon, B.Uguen \\ IETR, University of Rennes 1 \\ \{mohamed.laaraiedh, bernard.uguen, stephane.avrillon\}@univ-rennes1.fr
}

\begin{abstract}
In this paper, localization based on Received Signal Strength (RSS) is investigated assuming a path loss log normal shadowing model. On the one hand, indirect RSS-based estimation schemes are investigated; these schemes are based on two steps of estimation: estimation of ranges from RSS and then estimation of position using weighted least square approximation. We show that the performances of this type of schemes depend on the used estimator in the first step. We suggest that typical median estimator must be replaced by maximum likelihood estimator (mode) to enhance the positioning accuracy. On the other hand, a new direct RSS-based estimation scheme of position is proposed; Monte Carlo simulations show that the new estimator performs better than indirect estimators and can be reliable in future hybrid localization systems.
\end{abstract}

Index Terms-Localization, RSS, Indirect vs Direct Location Estimation, Weighted least square, Hybrid Data Fusion, 4G networks, ranging, Path Loss, Log Normal Shadowing.

\section{INTRODUCTION}

Nowadays, Location Based Services (LBSs) are more and more required by people and industries. Security is the main motivation for civilian mobile position location whose implementation is nowadays mandatory for the emergency calls. Besides security, the second leading application for wireless localization is intelligent transportation systems (ITSs). Personal tracking, navigation assistance and positiondependent billing are also new LBSs in expansion [1]. Furthermore, the location information is not only valuable for itself to provide new services but also to improve cellular communication systems at various levels. This is the scope of the FP7 WHERE project [2].

Location methods based on Received Signal Strength (RSS) have an important advantage compared with others methods since RSS is usually available whatever is the Radio Access Network (RAN) [3]. Nevertheless, the precision and accuracy of RSS is different from one RAN to another. The challenge here is to merge hybrid RSSs characterized by different accuracies and coming from different systems in order to enhance the position accuracy. In the following, hybrid RSS fusion relates to an algorithm which make use of RSS observables coming from different RANs (Cellular, WLAN, UWB, etc). This is the typical case in $4 \mathrm{G}$ networks where nodes with different technological platforms are integrated and in which the MS may be connected conjointly to cellular Base Station (BS) and wireless Access Point (AP) [4].
Historically, RSS can be used in either fingerprinting or lateration. Fingerprinting with RSS refers to the type of algorithms that first collect RSS fingerprints of a scene and then estimate the location of the MS by matching on-line measurements with the closest location fingerprints [5]. RSS lateration consists in estimating the ranges from collected RSSs assuming a path loss model and then computing position using these different estimated ranges. Generally, to estimate range from RSS the median estimator is used [6], [7], [8], [9]. This estimator do not require the knowledge of shadowing which affects the RSS measurements, and it is useful when no information about shadowing is available. Nevertheless, in the case of a non Gaussian distribution, this estimator performs worse than the Maximum Likelihood estimator (ML) given by the mode of the distribution.

In the present study, we distinguish the indirect from the direct schemes of positioning with RSS. The indirect schemes are commonly used in previous works and consists in positioning using ranges previously estimated from RSS measurements assuming a path loss model. The proposed direct approach consists in the estimation of position directly from RSS measurements without going through ranges. Assuming a log normal shadowing model for path loss, three indirect estimators (mean, median and mode) are firstly investigated; Then, the new direct estimation scheme is proposed. These different estimators are evaluated by Monte Carlo simulations and show that mode estimator is the best indirect estimator and that the new direct estimator performs better than all direct schemes.

The rest of the paper is organized as follows. Section II investigates the log normal shadowing model and presents the different radio propagation parameters which may affect the positioning accuracy. Section III presents the three indirect estimation schemes. Then, section IV proposes the new direct estimation scheme and its mathematical formulation. In section $\mathrm{V}$, the performances of each estimator are evaluated and discussed using Monte Carlo simulations. Finally, our concluding remarks are given in section VI.

In order to simplify the lecture of this paper, a list of abbreviations and symbols that are used in the paper is given in Table I. 


\begin{tabular}{ll}
\hline AN & Anchor Node \\
LS & Least Square \\
ML & Maximum Likelihood \\
MS & Mobile Station \\
RSS & Received Signal Strength \\
$d$ & Distance between transmitter and receiver (m) \\
$d_{0}$ & Reference distance generally equal to 1 meter \\
$L$ & Pathloss at distance $d(\mathrm{~dB})$ \\
$L_{0}$ & Pathloss at distance $d_{0}(\mathrm{~dB})$ \\
$n_{p}$ & Pathloss exponent \\
$\lambda$ & Wavelength (m) \\
$\sigma_{s h}$ & Standard Deviation of shadowing (dB) \\
$\mathbf{x}=(x, y)$ & Coordinates of the MS \\
$\mathbf{x}_{k}=\left(x_{k}, y_{k}\right)$ & Coordinates of the $k^{t h}$ AN \\
$l$ & Length of the simulated area \\
$N_{\text {Trial }}$ & Number of Trials in Monte Carlo simulations \\
\hline
\end{tabular}

TABLE I: List of different used abbreviations and symbols. table

\section{Log Normal Shadowing Path Loss Model}

The simple analysis often used in coexistence studies limits the propagation characteristics to the large scale of the signal at given distances (pathloss). In mathematical terms, the mean received power (around which there will still be shadowing and multipath) will vary with distance with an exponential law. The total pathloss at a distance, $d$, will then be L, often modelled as [10]:

$$
L=L_{0}+10 n_{p} \log \left(\frac{d}{d_{0}}\right)
$$

$d_{0}, d, n_{p}$ and $L_{0}$ are defined in Table I. $L_{0}$ is given by:

$$
L_{0}=20 \log \left(\frac{4 \pi d_{0}}{\lambda}\right)
$$

In fact this expression of $L$ represent only the mean loss of the power. The measured loss varies about this mean according to a zero-mean Gaussian random variable, $X_{\sigma_{s h}}$, with standard deviation $\sigma_{s h}$. Shadowing is caused by obstacles between the transmitter and receiver that attenuate signal power through absorption, reflection, scattering, and diffraction. The complete path loss equation expressed in $\mathrm{dB}$ is then given by:

$$
L=L_{0}+10 n_{p} \log \left(\frac{d}{d_{0}}\right)+X_{\sigma_{s h}}
$$

This model can be used for both indoor and outdoor environments. For each environment or/and radio link, a characteristic value of each parameter, $n_{p}$ and $\sigma_{s h}$, is used. These values can be determined by calibration via measurement companions. Furthermore, the frequency and the bandwidth affect these parameters. The most common values of $n_{p}$ are shown by Table II for different types of environments.

\begin{tabular}{ll}
\hline Type of environment & Path loss exponent $n_{p}$ \\
\hline Free Space & 2 \\
Urban area cellular radio & 2.7 to 3.5 \\
Shadowed urban cellular radio & 3 to 5 \\
In building LOS & 1.6 to 1.8 \\
Obstructed in building & 4 to 6 \\
Obstructed in factory & 2 to 3 \\
\hline
\end{tabular}

TABLE II: Path Loss Exponent for different environments [4]. table
The log normal shadowing model is very interesting for localization because it defines a linear relation between RSS and the logarithm of the distance between MS and AN. Nevertheless, the precision of estimated distance decreases as the separation between MS and AN increases. As a rule of thumb, if $n_{p}=2$ then RSS drops by $6 \mathrm{~dB}$ every time distance doubles. This sub-linear attenuation rate means that the difference in RSS between $1 \mathrm{~m}$ and $2 \mathrm{~m}$ is similar to the difference between $10 \mathrm{~m}$ and $20 \mathrm{~m}$ : exactly $6 \mathrm{~dB}$ (Fig. 1). Taking this into account, a constant level of noise can result in ever increasing error when RSS is used to estimate distance; if RSS noise is sufficient that we cannot tell the difference between 1 and $1.5 \mathrm{~m}$, we also cannot tell the difference between $10 \mathrm{~m}$ and $15 \mathrm{~m}$. As shown in Fig. 1, changes in RSS due to distance become small relative to noise, even if the level of noise remains the same over distance [11].

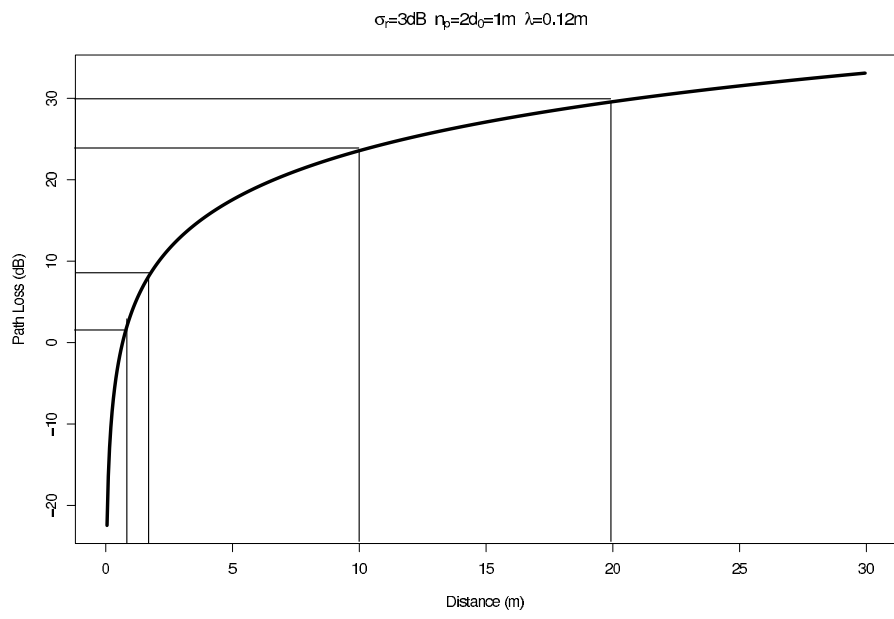

Fig. 1: Variation of path loss with respect to distance using Log Normal Shadowing model: Error increases over distance depending on both noise and attenuation rate. As the path loss flattens out, differences in RSS become small relative to noise level. figure

\section{RSS-BASED INDIRECT ESTIMATORS}

In this section, we investigate the indirect RSS-based positioning schemes which consist in two steps: estimation of ranges from RSS observables and then estimation of position using weighted LS approximation on the previously estimated ranges

\section{A. Estimation of range from RSS}

Let's consider the log normal shadowing described by the equation (3) as the used path loss model where we assume that the shadowing term $X_{\sigma_{s h}}$ is zero-mean Gaussian :

$$
X_{\sigma_{s h}} \sim \mathcal{N}\left(0, \sigma_{s h}^{2}\right)
$$

From (3) and (4) we derive the fact that the distance $d$ follows a Log-Normal distribution :

$$
p_{d}(d, L)=\frac{1}{\sqrt{2 \pi} d S} e^{\frac{-(\ln d-M)^{2}}{2 S^{2}}}
$$


where

$$
\begin{gathered}
S=\frac{\sigma_{s h} \ln 10}{10 n_{p}} \\
M=\frac{\left(L-L_{0}\right) \ln 10}{10 n_{p}}+\ln d_{0}
\end{gathered}
$$

As $d$ follows a Log-Normal distribution, the mean, median and mode of estimated distance $\hat{d}$ are given respectively by [12]:

$$
\begin{aligned}
& \hat{d}_{L S}=e^{M+\frac{S^{2}}{2}} \\
& \hat{d}_{\text {median }}=e^{M} \\
& \hat{d}_{M L}=e^{M-S^{2}}
\end{aligned}
$$

From equations (8) to (10), one can notice that the only estimator that does not consider the knowledge of shadowing, given by the term $S$, is the median. Thus, this estimator may be practical when no information about shadowing is available. Once the MS get this knowledge, the best estimator will be the mode which is the ML estimator. The mean estimator is not a good choice as it over estimates the distance, and it is very inaccurate especially for strong values of $S$.

To better evaluate the performances of these different estimators, we derived for each estimator its variance. We obtained the estimated variances of mean, median, and mode estimators of distance are, respectively, given by:

$$
\begin{gathered}
\hat{\sigma}_{L S}^{2}=\hat{d}_{L S}^{2} e^{2 S^{2}}\left(e^{S^{2}}-1\right)=e^{2 M+3 S^{2}}\left(e^{S^{2}}-1\right) \\
\hat{\sigma}_{\text {median }}^{2}=\hat{d}_{\text {median }}^{2} e^{S^{2}}\left(e^{S^{2}}-1\right)=e^{2 M+S^{2}}\left(e^{S^{2}}-1\right) \\
\hat{\sigma}_{M L}^{2}=\hat{d}_{M L}^{2}\left(1-e^{-S^{2}}\right)=e^{2 M-2 S^{2}}\left(1-e^{-S^{2}}\right)
\end{gathered}
$$

\section{B. Weighted LS estimation of position}

Once the MS gets the necessary amount of RSS observables (3 at least in 2D scenario), it can perform the first step by estimating the different ranges $\left(\hat{d}_{k}\right)_{k=1, \ldots, K}$ with respect to the $K$ discovered AN in the scene. These ranges can be estimated using one of the three estimators given by (8), (9) or (10). Thus, we obtain the system :

$$
\begin{gathered}
\left(x-x_{1}\right)^{2}+\left(y-y_{1}\right)^{2}=\hat{d}_{1}^{2} \\
\ldots \\
\left(x-x_{K}\right)^{2}+\left(y-y_{K}\right)^{2}=\hat{d}_{K}^{2}
\end{gathered}
$$

Subtracting the first one $(k=1)$ from others equations of (14) results in

$$
2\left[\begin{array}{cc}
x_{2}-x_{1} & y_{2}-y_{1} \\
\ldots & \ldots \\
x_{K}-x_{1} & y_{K}-y_{1}
\end{array}\right]\left[\begin{array}{l}
x \\
y
\end{array}\right]=\left[\begin{array}{c}
h_{2}^{\prime}+\hat{d}_{1}^{2}-\hat{d}_{2}^{2} \\
\ldots \\
h_{K}^{\prime}+\hat{d}_{1}^{2}-\hat{d}_{K}^{2}
\end{array}\right]
$$

where $h_{k}^{\prime}=x_{k}^{2}-x_{1}^{2}+y_{k}^{2}-y_{1}^{2}$ for $k$ in $(2, . ., K)$.

The least square solution is then given by [6]:

$$
\mathbf{x}=\frac{1}{2}\left(\mathbf{A}^{T} \mathbf{A}\right)^{-1} \mathbf{A}^{T} \mathbf{h}
$$

where

$$
\begin{gathered}
\mathbf{A}=\left[\begin{array}{cc}
x_{2}-x_{1} & y_{2}-y_{1} \\
\ldots & \cdots \\
x_{K}-x_{1} & y_{K}-y_{1}
\end{array}\right], \mathbf{x}=\left[\begin{array}{l}
x \\
y
\end{array}\right] \\
\mathbf{h}=\left[\begin{array}{c}
h_{2}^{\prime}+\hat{d}_{1}^{2}-\hat{d}_{2}^{2} \\
\ldots \\
h_{K}^{\prime}+\hat{d}_{1}^{2}-\hat{d}_{K}^{2}
\end{array}\right]
\end{gathered}
$$

In order to enhance the performances of LS regression, we introduce the matrix of covariance of estimated ranges. Three covariance matrices are then defined depending on used ranges estimator. For the mean, median and mode estimator, respetively, this covariance matrix is given by:

$$
\begin{aligned}
\mathbf{R}_{L S} & =\operatorname{diag}\left(\left(\hat{\sigma}_{L S, k}^{2}\right)_{k=2, \ldots, K}\right) \\
\mathbf{R}_{\text {median }} & =\operatorname{diag}\left(\left(\hat{\sigma}_{\text {median }, k}^{2}\right)_{k=2, . ., K}\right) \\
\mathbf{R}_{M L} & =\operatorname{diag}\left(\left(\hat{\sigma}_{M L, k}^{2}\right)_{k=2, . ., K}\right)
\end{aligned}
$$

The weighted least square solution is then given by [6]:

$$
\mathbf{x}=\frac{1}{2}\left(\mathbf{A}^{T} \mathbf{R}^{-1} \mathbf{A}\right)^{-1} \mathbf{A}^{T} \mathbf{R}^{-1} \mathbf{h}
$$

where $R$ can be $R_{L S}, R_{\text {median }}$, or $R_{M L}$.

\section{PROPOSED RSS-BASED DIRECT ESTIMATOR}

In this fourth section, the mathematical formulation of the proposed direct estimation scheme is described. To proceed, let's assume that the MS is connected to $K$ ANs. For each link $k$, the distribution of $d_{k}=\left\|\mathbf{x}-\mathbf{x}_{k}\right\|$ is given by equation (5):

$$
\begin{aligned}
p_{k}\left(\left\|\mathbf{x}-\mathbf{x}_{k}\right\|, L_{k}\right) & =\frac{1}{\sqrt{2 \pi}\left\|\mathbf{x}-\mathbf{x}_{k}\right\| S_{k}} e^{\frac{-\left(\ln \left\|\mathbf{x}-\mathbf{x}_{k}\right\|-M_{k}\right)^{2}}{2 S_{k}^{2}}} \\
& =\frac{1}{\sqrt{2 \pi} d_{k} S_{k}} e^{\frac{-\left(\ln d_{k}-M_{k}\right)^{2}}{2 S_{k}^{2}}}
\end{aligned}
$$

In order to simplify the study, we assume the independence of the $K$ random variables $\left(p_{d_{k}}\right)_{k=1, . . K}$. Hence, the conjoint probability density function of these $K$ random variables is given by :

$p_{1 \ldots K}\left(d_{1} \ldots d_{K}, L_{1} \ldots L_{K}\right)=\prod_{k=1}^{K} \frac{1}{\sqrt{2 \pi} d_{k} S_{k}} e^{\frac{-\left(\ln d_{k}-M_{k}\right)^{2}}{2 S_{k}^{2}}}$

Let's introduce $F(\mathbf{x})=\ln \left(p_{1 \ldots K}\left(d_{1} \ldots d_{K}, L_{1} \ldots L_{K}\right)\right)$. Thus $F$ is given by:

$$
F(\mathbf{x})=-\sum_{k=1}^{K}\left(\ln \left(\sqrt{2 \pi} d_{k} S_{k}\right)+\frac{\left(\ln d_{k}-M_{k}\right)^{2}}{2 S_{k}^{2}}\right)
$$

The proposed new ML estimator is then defined by:

$$
\hat{\mathbf{x}}=\min _{\mathbf{x}} F(\mathbf{x})
$$


Developing the expression of $F$ leads to:

$$
\hat{\mathbf{x}}=\min _{x} F(\mathbf{x})=\min _{\mathbf{x}} \sum_{k=1}^{K}-\frac{\left(\ln \left\|\mathbf{x}-\mathbf{x}_{k}\right\|-\left(M_{k}-S_{k}^{2}\right)\right)^{2}}{2 S_{k}^{2}}
$$

To minimize $F$, we derived its gradient $\nabla F$. It can be readily shown then that the proposed ML estimator follows the implicit relation given by:

$$
\nabla F(\hat{\mathbf{x}})=\sum_{k=1}^{K} \frac{1}{S_{k}^{2}} \frac{\left(\left(M_{k}-S_{k}^{2}\right)-\ln \left\|\hat{\mathbf{x}}-\mathbf{x}_{k}\right\|\right)}{\left\|\hat{\mathbf{x}}-\mathbf{x}_{k}\right\|} \frac{\hat{\mathbf{x}}-\mathbf{x}_{k}}{\left\|\hat{\mathbf{x}}-\mathbf{x}_{k}\right\|}=\mathbf{0}
$$

We remark that this function $\nabla F$ is ill-conditioned when $\left(M_{k}-S_{k}^{2}\right)$ is in $[0,1]$. In that case, the ML estimator may be given by:

$$
\nabla F(\hat{\mathbf{x}}) \cdot \hat{\mathbf{x}}=\mathbf{0}
$$

This functional (29) share with the previous one (28) the targeted position. However, we notice that it has an additional trivial solution at origin which hopefully can be easily eliminated if it comes out from the optimization algorithm.

\section{Simulations Results and Discussions}

In this section, we evaluate the performances of the set of studied estimators described in section III and IV through Monte Carlo simulations. The different steps of the simulation are the following:

1) $K$ random ANs and one targeted MS are uniformly drawn in an area of $l \times l \mathrm{~m}^{2}$.

2) Different path losses $\left(L-L_{0}\right)$ are computed for each link $k$ between the MS and the $k^{t h}$ AN. For each link, log normal shadowing model is applied with appropriate $n_{p}, \lambda$ and $\sigma_{s h}$. Table III shows the used parameters for indoor and outdoor scenarios respectively.

3) The four different estimators are then evaluated for three different scenarios:

- Indoor.

- Outdoor.

- Indoor/Outdoor.

\begin{tabular}{lll}
\hline Parameters & Indoor & Outdoor \\
\hline$n_{p}$ & 1.6 to 1.8 & 2 to 4.0 \\
$\lambda(m)$ & 0.12 & 0.333 \\
$\sigma_{s h}(d B)$ & 2 to 5 & 2 to 5 \\
$l(m)$ & 15 & 1000 \\
\hline
\end{tabular}

TABLE III: List of radio parameters used in simulations for both indoor and outdoor scenarios.

table

All simulations have been done with a number of trials equal to $N_{\text {Trial }}=300$. For each studied scenario, the correspondent figure (Fig. 2 to Fig. 4 respectively) compares the cumulative density functions of four estimation schemes with respect to the positioning error in order to suggest the best estimation scheme.
The Fig. 2 and Fig. 3 are obtained respectively for indoor and outdoor scenarios with the parameters described in Table III. These figures show that the indirect estimation scheme based on the mode estimator for ranges performs better than those usually used based on median and mean estimators. Moreover, these figures suggest that the new proposed direct estimator performs better than direct schemes in the two different cases (indoor and outdoor). Thus, we believe that the direct RSS-based estimation scheme of MS's position may enhances the positioning accuracy.

In order to show the reliability of this new direct estimator even in the case of hybrid RSS fusion, we carried simulations in a typical $4 \mathrm{G}$ scenario where the MS can be connected conjointly to cellular BSs and wireless APs. The Fig. 4 shows the performances of different estimators for this scenario with $l=1000 \mathrm{~m}$. This figure is obtained by reproducing the same simulations conditions assumed in Fig. 3 but with adding two indoor links into a square of $l=15 \mathrm{~m}$. The position of MS is chosen randomly in the sqaure $15 \times 15 \mathrm{~m}^{2}$. This is done by respecting the different assumed parameters $\left(n_{p}, \lambda\right.$ and $\left.\sigma_{s h}\right)$ for indoor scene given by Table III for each additional link. This figure emphasizes the expected conclusions and shows that the new proposed estimator enhances the performances of hybrid RSS-based localization.

Comparison between Fig. 3 and Fig. 4 shows that the enhancement performed by the direct estimator, after adding indoor links, is major than the enhancement performed in the case of indirect estimators. These first constatations suggest that the proposed direct estimator is more reliable when hybrid RANs are used. Furthermore in this type of scenarios, estimators may experience short and long range links at the same time. In this case, the precisions of estimated distances from RSS observables can be very different as explained in Fig. 1. We believe that the direct estimator is not influenced by these imprecisions because it uses RSS observables directly without going through ranges estimations.

\section{CONCLUSION}

In this paper, we studied hybrid RSS-based localization estimators assuming a path loss log normal shadowing model. We distinguished direct from indirect schemes. Indirect estimation schemes consist in two steps: estimation of ranges from RSS using mean, median or mode estimators; and estimation of location using weighted least square approximation on previously estimated ranges. We showed that estimation of ranges from RSS and consequently positioning accuracy can be enhanced using mode estimator rather than median or mean estimators usually used in past studies. Furthermore, a new direct scheme for location estimation from RSS is proposed and analyzed. This new estimator performs better than indirect schemes and may enhances positioning accuracy using hybrid RSS observables coming from different radio access networks. Next step will be to evaluate performance in more realistic scenarios and especially by using more realistic path loss model with adequate parameters. 


\section{ACKNOWLEDGMENT}

The work presented in this paper has been performed in the framework of the ICT-217033 WHERE project funded by the European Union.

\section{REFERENCES}

[1] P. Bellavista, A. Kupper, and S. Helal, "Location-based services: Back to the future," IEEE, Pervasive Computing, 2008.

[2] "http://www.kn-s.dlr.de/where/.",

[3] H. Laitinen, S. Juurakko, T. Lahti, R. Korhonen, and J. Lahteenmaki, "Experimental evaluation of location methods based on signal-strength measurements," IEEE transactions on vehicular technology, vol. 56, Jan. 2007.

[4] A. Goldsmith, Wireless communications. 2005.

[5] H. Liu, H. Darabi, P. Banerjee, and J. Liu, "Survey of wireless indoor positioning techniques and systems," IEEE Transactions on systems, man, and cybernetics, vol. 37, Nov. 2007.

[6] K. Cheung, H. So, W. Ma, and Y. Chan, "A constrained least squares approach to mobile positioning: Algorithms and optimality," 2006.

[7] T. Gigl, G. J. M. Janssen, V. Dizdarevic, K. Witrisal, and Z. Irahhauten, "Analysis of a uwb indoor positioning system based on received signal strength," WPNC 07, 2007.

[8] M. Sugano and T. Kawazoe, "Indoor localization system using rssi measurement of wireless sensor network based on zigbee standard," WSN 06, July 2006

[9] S. Frattasi, M. Monti, and P. Ramjee "A cooperative localization scheme for $4 \mathrm{~g}$ wireless communications," IEEE Radio and Wireless Symposium, 2006.

[10] V. Abhayawardhana, W. Crosby, M. Sellars, and M. Brown, "Comparison of empirical propagation path loss models for fixed wireless access systems," IEEE VTC spring, 2005.

[11] K. Whitehouse, C. Karlof, and D. Culler, "A practical evaluation of radio signal strength for ranging-based localization," Mobile Computing and Communications Review, vol. 11, no. 1, 2007.

[12] M. P.McLaughlin, A Compendium of Common Probability Distributions, vol. Regress+ Documentation. 1999

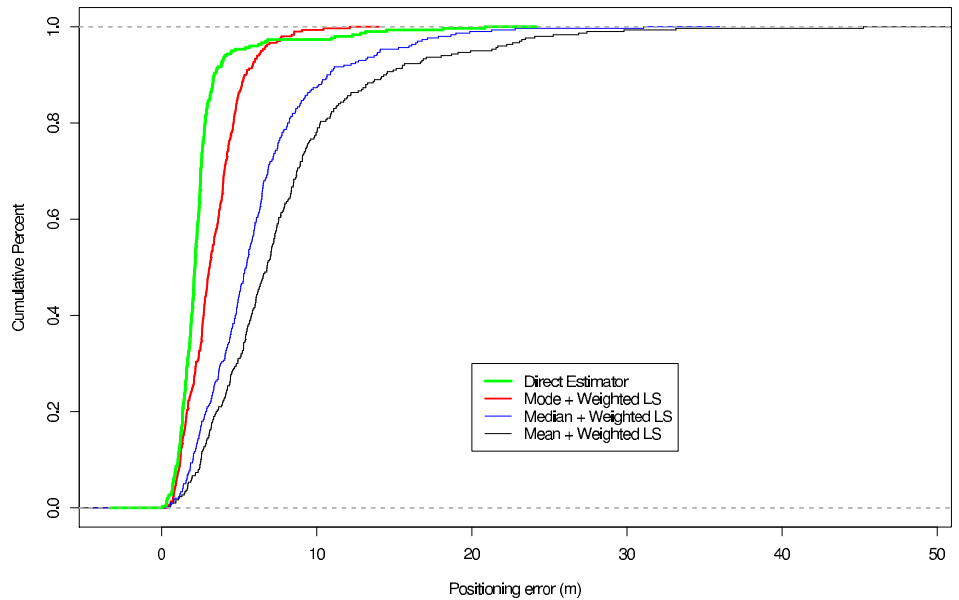

Fig. 2: Compared cumulative density function of four studied estimators for indoor scenario $-l=15 \mathrm{~m}, K=5$. figure

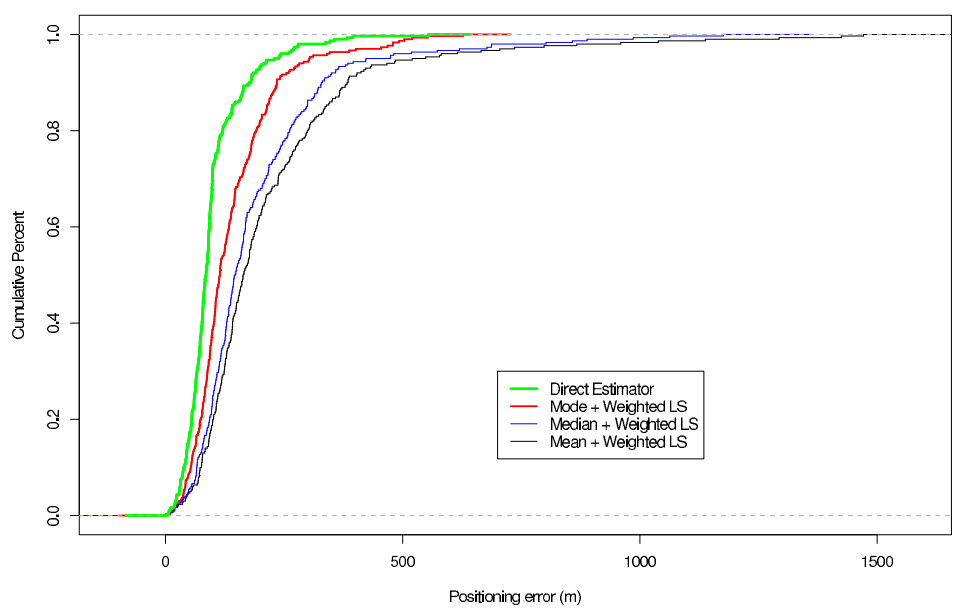

Fig. 3: Compared cumulative density function of four studied estimators for outdoor scenario - $l=1000 \mathrm{~m}, K=5$. figure

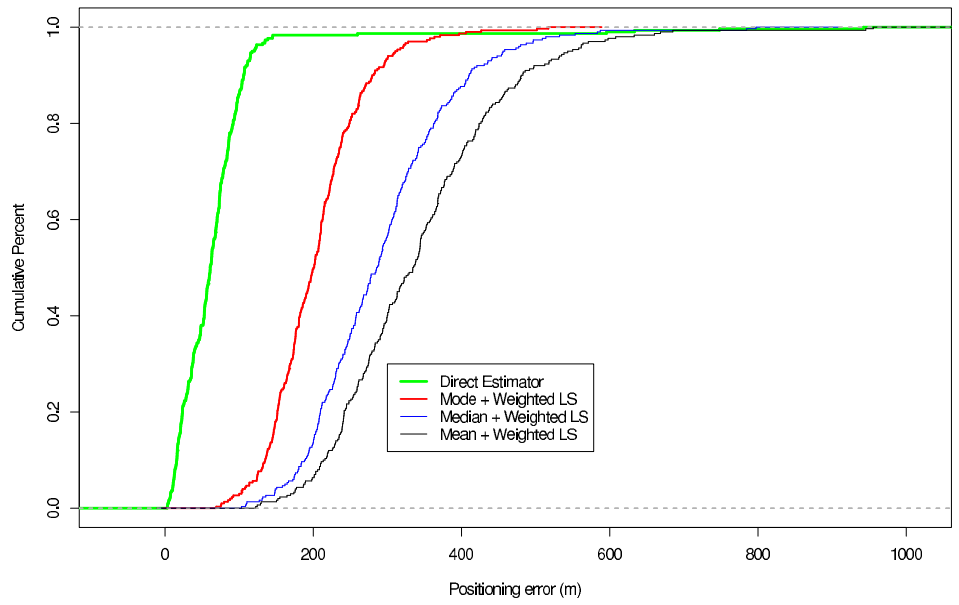

Fig. 4: Compared cumulative density function of four studied estimators for hybrid scenario $-l=1000 m, K=5 B S+2 A P$. figure 